\title{
Disaggregation von Bevölkerungsdaten - Probleme und Lösungsansätze am Beispiel der Agglomeration Zürich
}

\section{Einleitung}

Für die meisten Analysen mit anthropogeographischem Inhalt spielt die zahlenmäßige Größe und Verteilung der Bevölkerung eine zentrale Rolle. Ganz besonders gilt dies für Standort- und Lageanalysen, bei denen bereits Verschiebungen um wenige hundert Meter große Auswirkungen auf die Zahl der «betroffenen» bzw. «erreichbaren» Haushaltungen haben können. Dazu gehören beispielsweise die Planung von Infrastruktureinrichtungen oder die Ausbreitung von Emissionen. Der vorliegende Artikel ist das Resultat der Anwendung von Instrumenten, wie sie am Geographischen Institut der Universität Zürich im Laufe von kleinräumigen Untersuchungen in der Agglomeration und im Kanton Zürich entwickelt wurden. Da nicht auf vorhandene Methoden zurückgegriffen werden konnte, weisen die «Problemlösungen» notgedrungen einen stark empirischen Charakter auf.

Die 81 Gemeinden, die zusammen die Aggolomeration Zürich bilden, weisen bezüglich der Bevölkerungszahl sehr unterschiedliche Größenordnungen auf. Das Verhältnis der Einwohnerzahl zwischen der größten (Stadt Zürich) und der kleinsten $\mathrm{Ge}$ meinde (Islisberg) betrug bei derVolkszählung 1980 $1: 2350$. Für den Kanton Zürich lautet das Verhältnis $1: 1800$ (Stadt Zürich bzw. Volken). Die Resultate einer vergleichenden Analyse, die nur mit Daten auf Gemeindeebene arbeitet, ist wegen der unterschiedlichen Informationsdichte für die meisten Fragestellungen ungenügend, im schlechtesten Fall sogar irreleitend. In die letzte Kategorie gehört zum Beispiel die Versorgung mit Arzt- oder Zahnarztpraxen, wo der hohen Angebots-Dichte in einigen Innenstadtquartieren eine eigentliche Unterversorgung in vielen Stadtrandquartieren gegenübersteht. Bei einer Verwendung der Einteilung der Stadt $\mathrm{Zü-}$ rich in 34 Quartiere läßt sich das Verhältnis zwischen bevölkerungsmäßig größter und kleinster Einheit auf rund $1: 175$ bzw. $1: 135$ reduzieren. Da die meisten dieser Quartiere aus einst selbständigen Gemeinden hervorgegangen sind, ergibt sich auch im Kartenbild eine - prinzipiell wünschbare - Angleichung der Größenverhältnisse. Oft sind jedoch auch diese 114 Einheiten in sich zu heterogen und zu wenig detailliert für eine präzise Aussage.

\section{Vorhandene Datenquellen mit kleinräumigem Bezugsniveau}

Einheitliche kleinräumige Gliederungen, wie sie z. B. in der Bundesrepublik Deutschland im Zusammenhang mit den Wahlkreisen bestehen, gibt es in der Schweiz nicht. Eigentlich könnten die Zählkreise der Volkszählungen diese Funktion ausüben, doch sind Pläne mit den Grenzen dieser Zählkreise nur in Einzelfällen noch greifbar. Außerdem unterliegt die bei jeder Volkszählung anders durchgeführte Abgrenzung der Zählkreise mit Ausnahme einer größenmäßigen Beschränkung auf etwa 100 bis 150 Haushaltungen keinerlei Homogenitätskriterien. Eine Auswertung auf kleinräumiger Ebene muß sich daher anderer Quellen bedienen. Eine fast ideale, weil informationsreiche, gut vergleichbare und für quantitative Analysen besonders geeignete Datenbasis ist die hektarweise Erfassung von Bevölkerungsdaten. In der Schweiz wurde diese Methode anläßlich der Volkszählungen 1970 und 1980 vom Bundesamt für Statistik empfohlen. Aufgrund des föderalistischen Staatsaufbaus lag der Entscheid über die Durchführung bei den einzelnen Kantonen und Gemeinden. In der Folge führten 1970755 von insgesamt 3063 Gemeinden eine hektarweise Codierung der Volkszählungsfragebogen durch; 1980 waren es 620 von 3029 Gemeinden. Dabei wurde nur in Ausnahmefällen eine Flächendeckung für größere Gebiete erreicht. Auf die Agglomeration Zürich übertragen bedeutet dies, daß für 40 von $81 \mathrm{Ge}$ meinden mit rund $75 \%$ aller Einwohner Daten auf Hektarbasis erhoben wurden (vgl. Abb. 1). Für den Kanton Zürich sind es 66 von 171 Gemeinden mit $71 \%$ aller Einwohner.

Eine auf diesen Rasterdaten aufbauende Analyse hat nur einen Sinn, wenn es gelingt, die durch die fehlenden Gemeinden bedingten "weißen Flecken» möglichst wahrheitsgetreu zu füllen. Für diesen Zweck bietet sich die Siedlungsstatistik an, die für

Matthias Bopp, dipl. Geograph und

Adrian Herzog, dipl. Geograph,

Geographisches Institut, Universität Zürich-Irchel, Winterthurerstr. 190, 8057 Zürich 


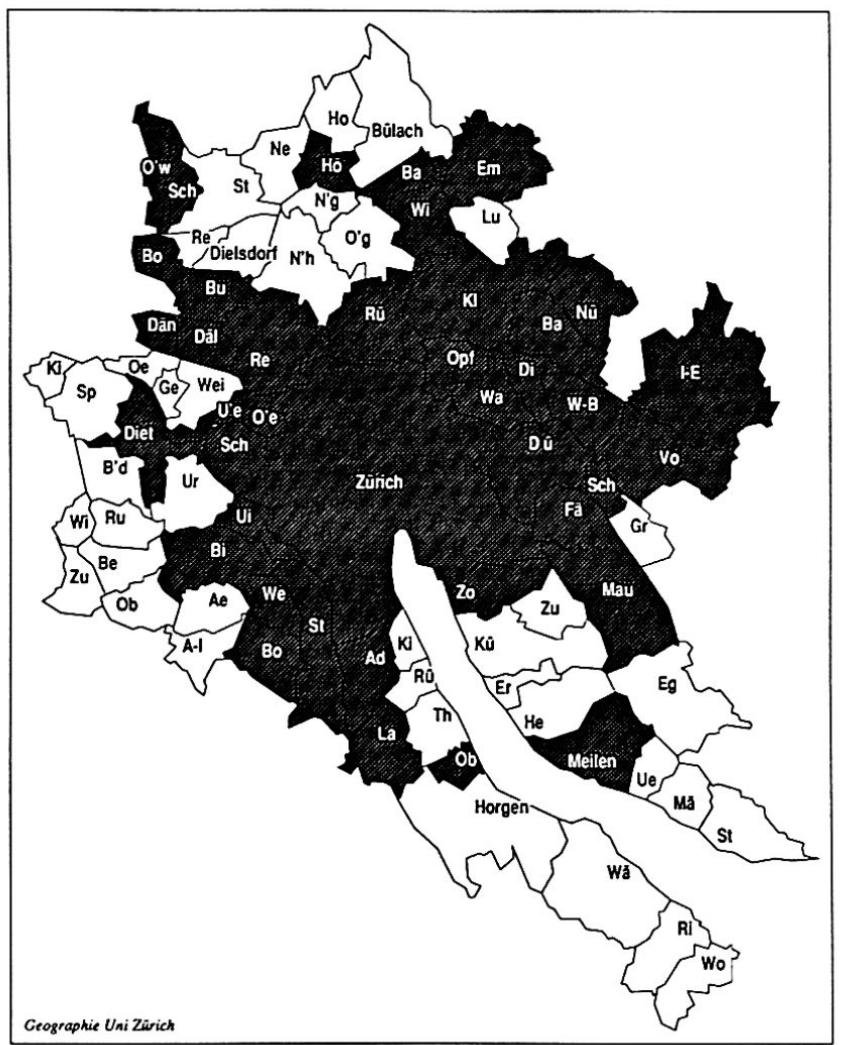

Abb. 1 Gemeinden der Agglomeration Zürich

Gemeinden mit hektarweiser Aufschlüsselung der Volkszählung 1980 sind dunkel dargestellt, die übrigen hell

den Kanton Zürich seit 1950 bei jeder Volkszählung erstellt wird (STATISTISCHES AMT DES KANTONS ZÜRICH, 1985, S. I).

Die Siedlungsstatistik arbeitet mit dem Siedlungsbegriff nach WINDLER und WINKLER': "Eine Siedlung ist eine Einzelwohnstätte oder eine als zusammenhängendes Ganzes erscheinende Gruppe von nahe zusammenstehenden Wohngebäuden. Siedlungen zeigen eine gewisse räumliche Geschlossenheit, einen räumlichen Zusammenhang, aber auch eine deutliche Abgrenzung gegenuiber anderen Siedlungen. Eine Stadt, ein Dorf, ein Weiler oder auch ein bewohnter Einzelhof stellen demnach Siedlungen dar.»

Bei der Abgrenzung durch das Statistische Amt des Kantons Zürich wurden Wohngebäude in der Regel zu einer Siedlung zusammengefaßt, falls sie weniger als 100 Meter voneinander entfernt waren. Als weitere trennende oder verbindende Kriterien wurden Höhenunterschiede, Hindernisse und Verkehrsmöglichkeiten berücksichtigt. Einige der so abgegrenzten Siedlungen mußten benachbarten Siedlungen zugeschlagen werden, weil sie entweder keinen Eigennamen besitzen oder von den Gemeindebehörden nicht als selbständige Siedlungen anerkannt wurden (ebenda, S.2). Im weiteren gelten die Kriterien zur Siedlungsabgrenzung nur innerhalb des jeweiligen Gemeindegebietes, d. h. es werden auch bei völlig zusammengewachsenen Gemeinden keine die Gemeindegrenze überschreitenden Siedlungen gebildet. Insgesamt wurden für die Volkszählung 1980 auf dem Gebiet des Kantons Zürich 4595 Siedlungen unterschieden, wovon nur $119 \mathrm{mehr}$ als 1000 Einwohner zählten (ebenda, S. 3).

"Ziel der Siedlungsstatistik ist es, statistische Angaben für kleine geographische Räume bereitzustellen" (ebenda, S. 3). Umfangreiche Siedlungen sollten daher in Absprache mit den Gemeindebehörden nach dem Prinzip der Homogenität in Quartiere unterteilt werden. Da die praktische Durchführung jedoch Sache der einzelnen Gemeinden war, wurde die empfohlene Untergliederung der größeren Siedlungen in Quartiere sehr unterschiedlich gehandhabt. Von 66 Zürcher Gemeinden mit Siedlungen mit über 2000 Einwohnern (ohne die Städte Zürich und Winterthur, die eine rechtlich festgelegte Quartiereinteilung haben) haben nur 39 dieser Empfehlung nachgelebt (Agglomeration Zürich ohne Stadt: 31 von 49). Allerdings bleiben nur wenige größere Gemeinden, für die es weder eine hektarweise Auszählung noch eine brauchbare Einteilung in Ortsteile gibt.

\section{Vervollständigung der hektarweisen Auszählung unter Verwendung der Siedlungsstatistik}

\subsection{Vorarbeiten}

Zunächst wurden die Grenzen der Siedlungen und Quartiere aus Übersichtsplänen (meist im Maßstab $1: 5000$ ) digitalisiert. Dieser Schritt konnte mit einem Auftrag des Statistischen Amtes und des Amtes für Raumplanung des Kantons Zürich an das Geographische Institut der Universität Zürich kombiniert werden. Es zeigte sich allerdings bald, daß das Einzeichnen der Außengrenzen der Siedlungen wie ihre allfällige Untergliederung nach sehr uneinheitlichen Kriterien erfolgt sein mußte: Je nach Gemeinde gehören umfangreiche Industriegebiete und nicht überbaute Flächen (Friedhöfe, Schulanlagen usw.) ebenfalls zum Siedlungsgebiet, oder es wurde mit Akribie praktisch jedes unbewohnte Gebäude ausgeschlossen. Da der Kanton im Rahmen des Auftrags eine Umsetzung der Siedlungsstatistik in eine Bevölkerungsverteilung in einem $200-\mathrm{m}-\mathrm{Ra}$ ster wünschte (ohne Verwendung der Ergebnisse der hektarweisen Auszählung), wurden alle zu großzügig bemessenen Siedlungen neu digitalisiert. Dabei wurden alle über eine Hektare großen unbewohnten Flächen (Industrie- und Verkehrsanlagen, nicht überbaute Inseln im Siedlungsgebiet, Zweckbauten) ausgeschieden. Dadurch konnte das «Siedlungsgebiet» weitgehend auf die tatsächlich bewohnten Flächen reduziert werden (Abb. 2). Die so 
gewonnenen Umrisse sind für eine kleinmaßstäbliche kartographische Verwendung wenig geeignet, da sie ein sehr unruhiges Kartenbild mit vielen Inseln ergeben. Bei der angestrebten Disaggregation und Umsetzung von Bevölkerungsdaten von Polygon- auf Hektarbasis spielt dieser Umstand aber keine Rolle.

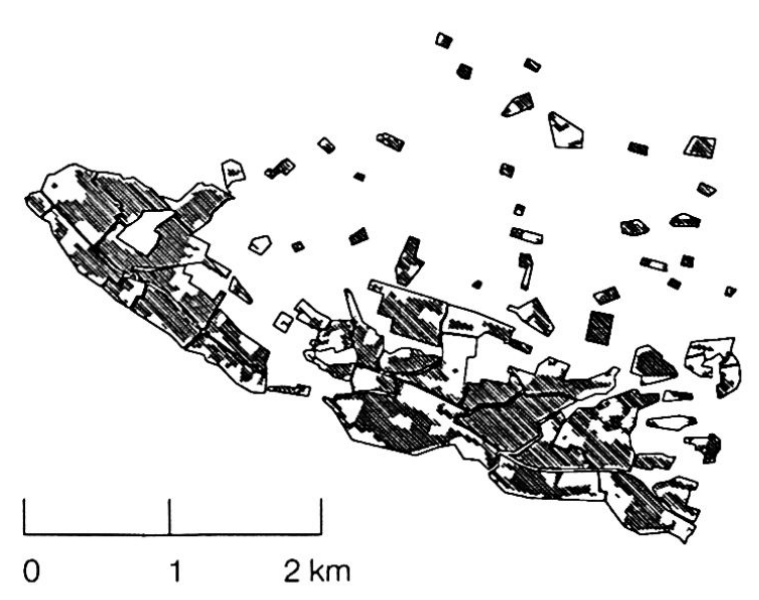

Abb. 2 Ursprüngliche und auf das eigentliche Wohngebiet reduzierte (schraffiert) Siedlungsumrisse am Beispiel der Gemeinde Meilen

\subsection{Umsetzung der Polygon- in Rasterinformation}

In Gemeinden ohne hektarweise Auszählung der Volkszählung kann nun mit Hilfe der so digitalisierten Siedlungsgebiete eine Schätzung der Bevölkerungsverteilung nach Hektaren versucht werden: Die Bevölkerung jeder Siedlung wird dabei gleichmäßig auf die dazugehörigen Hektaren verteilt. Um möglichst viel von der Information, die in den detailliert aufgenommenen Grenzlinienverläufen vorliegt, ins Raster hinüberzuretten, wurde eine verfeinerte Methode für den Rasterisierungsprozeß entwickelt: Zunächst wird für jede Siedlung ein zehnmal feinerer Raster (im vorliegenden Fall ein Raster mit einer Zellengröße von einer Are) aufgebaut, in dem die Siedlung vollständig Platz findet. Dann wird für den Zentrumspunkt jeder dieser Zellen festgelegt, ob er inner- oder außerhalb der bearbeiteten Siedlung liegt. Dabei wird die Beachtung von allfälligen Ex- und Enklaven durch einen Vorverarbeitungsschritt gewährleistet. Die auf die betreffende Siedlung zu verteilende Bevölkerungszahl wird anschließend durch die Zahl der innerhalb der Siedlung liegenden Aren geteilt. Diese durchschnittliche Bevölkerungszahl je Zelle des feinen Rasters wird nun für jede Zelle des groben Rasters (Hektaren) aufsummiert. Da die Wahrscheinlich- keit, daß nur marginal vom digitalisierten Siedlungsumriß angeschnittene Hektaren tatsächlich besiedelt sind, sehr gering ist, kann über einen einzugebenden Schwellenwert als Randbedingung eine minimale Bedeckung einer Rasterzelle durch das Siedlungsgebiet festgelegt werden. Dieser Prozeß wird nun für sämtliche Siedlungen einer Gemeinde durchgeführt und die Bevölkerungszahlen werden für jede Hektare bestimmt. In einem letzten Schritt werden die je Hektare aufsummierten Bevölkerungszahlen - unter Vermeidung von systematischen Fehlern - zu ganzen Zahlen gerundet und auf eine Datei ausgeschrieben. Die resultierende Rastermatrize kann nun mit den im Geographischen Informationssystem GRID/GRIDUNI verfügbaren Prozeduren weiter bearbeitet werden und beispielsweise statistisch analysiert oder kartographisch dargestellt werden.

\subsection{Bewertung der Resultate}

Eine Bewertung der geschilderten Methode kann durch optische und statistische Vergleiche sowie durch Überprüfungen anhand von für die Praxis typischen Fragestellungen erfolgen. Wir haben dafür als Beispiel die Gemeinde Meilen gewählt, weil hier neben der hektarweisen Auszählung eine ziemlich detaillierte Quartiereinteilung vorliegt. Obwohl die hektarweise Auszählung nicht völlig frei von Codierungsfehlern ist, und die Zuordnung der Gebäude und damit der Bewohner nicht streng der Geometrie der Rasterzellen folgen kann (ACKERKNECHT, 1969 , S. 41), ist sie zweifellos ein gutes Maß für die Qualität von Disaggregationsmethoden. Neben der oben beschriebenen Methode mit in den Vergleich einbezogen werden außerdem gleichförmige Verteilungen der Gemeindebevölkerung auf

- die gemäß Hektarauszählung bewohnten Rasterzellen,

- die Siedlungsgebiete und

- auf ein stark generalisiertes Siedlungsgebiet ohne Berücksichtigung der außerhalb gelegenen Kleinsiedlungen.

Weil kein direktes statistisches Maß für die Ähnlichkeit zwischen den verschiedenen Methoden existiert, wurde auf einige Hilfsgrößen zurückgegriffen. Obwohl die nicht normalverteilten Daten eine ungünstige Voraussetzung für einzelne Tests darstellen, bescheinigt Tabelle 1 der gewählten Disaggregationsmethode eine klare Überlegenheit über die anderen Methoden.

Noch besser ersichtlich wird die Ähnlichkeit zwischen Originalwerten und disaggregierten Werten im visuellen Vergleich (Abb. 3). Dieser Eindruck wird durch praktische Berechnungen mit den entsprechenden Rasterzellenwerten bestätigt (Tabelle 2, Situationskarte Abb. 4). 


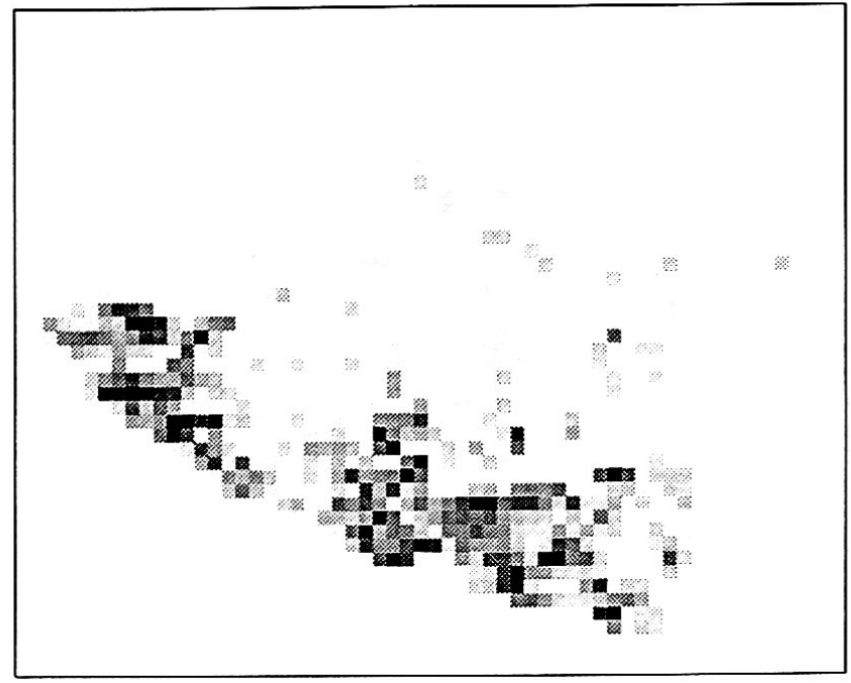

1: Idealfall (Originalwerte)

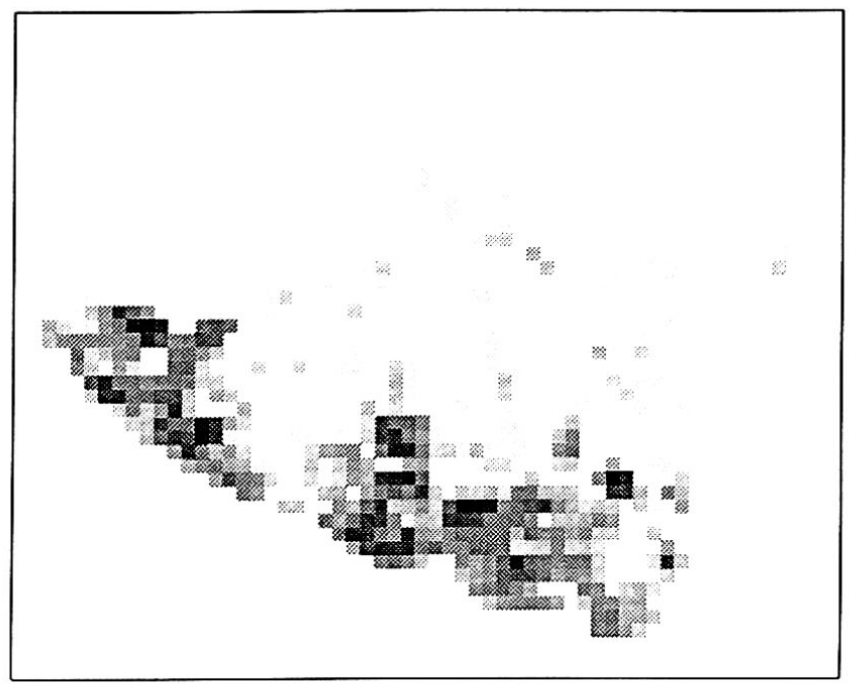

3: Siedlungsstatistik und -umrisse, Zellen mit unter 10\% Flächenanteil umverteil (vgl. Text)

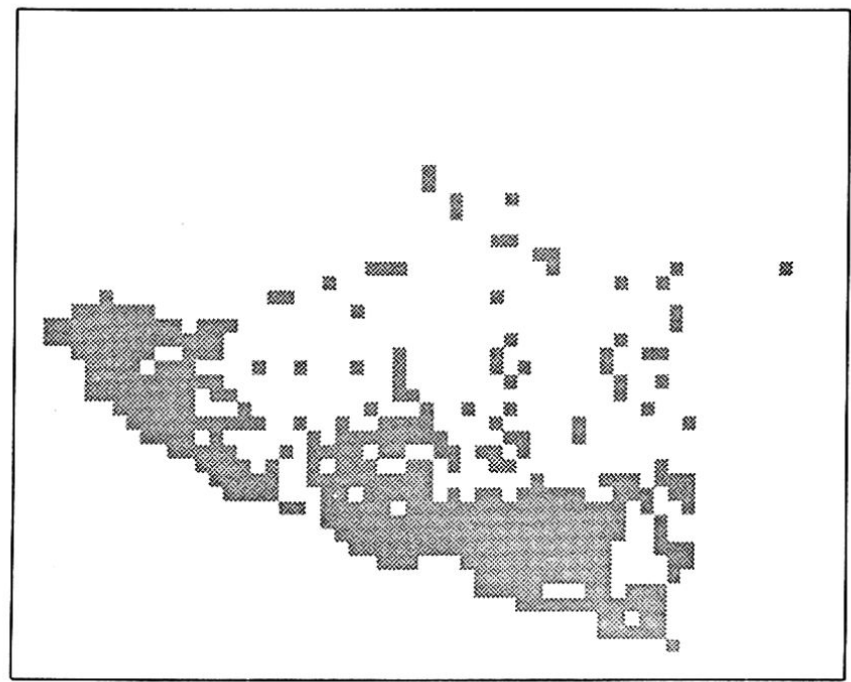

5: Bewohnte Zellen gemäß hektarweiser Auszählung, gleichförmige Verteilung der Bevölkerung

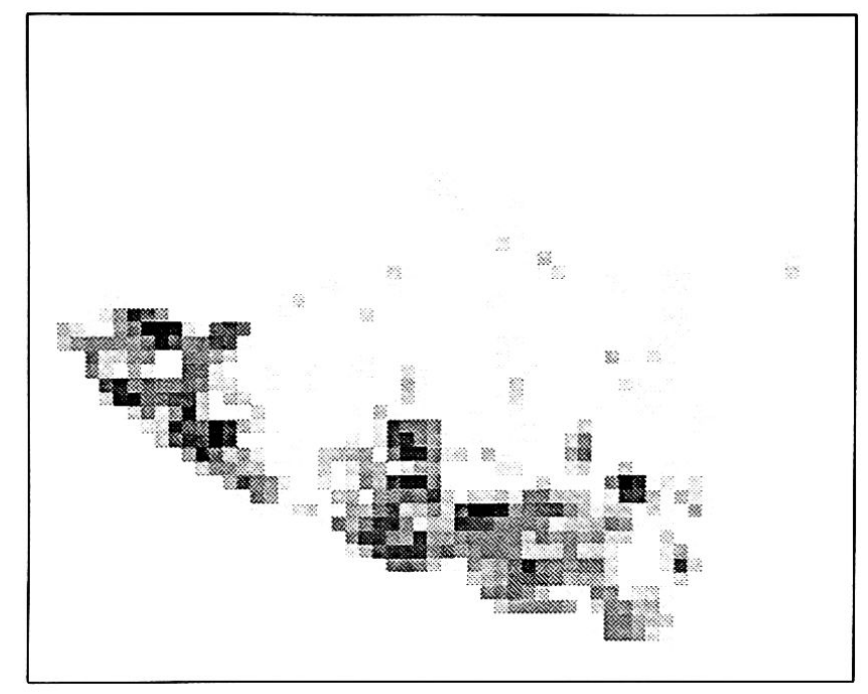

2: Siedlungsstatistik und -umrisse, alle Zellen

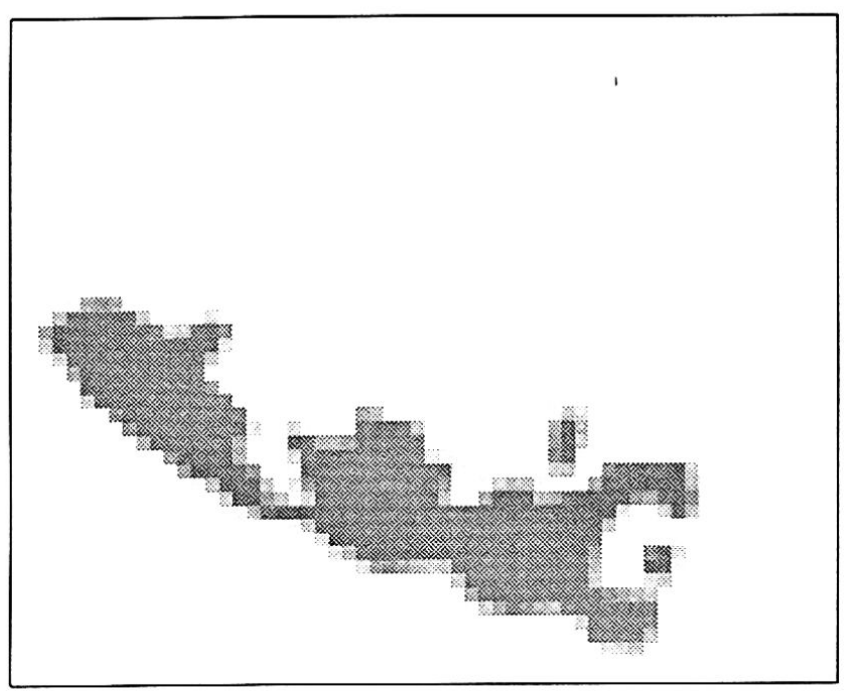

4: Siedlungsumrisse generalisiert, gleichförmige Verteilung der Bevölkerung

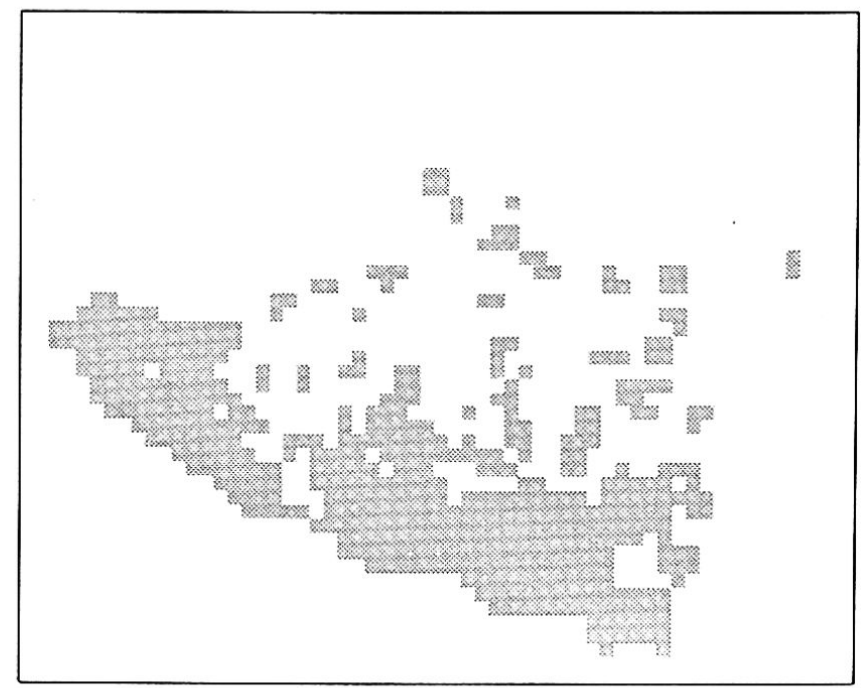

6: Siedlungsumrisse detailliert, gleichförmige Verteilung der Bevölkerung

Abb. 3 Bevölkerungsverteilung nach verschiedenen Disaggregationsmethoden (Beispiel Meilen) Der Grad der Schwärzung der einzelnen Rasterzellen ist proportional zur Bevölkerungszahl 
Tab. 1 Verschiedene Disaggregationsmethoden im statistischen Vergleich (Beispiel Meilen)

\begin{tabular}{llllrlrll} 
Methode & A & B & C & D & E & F & G & $H$ \\
\hline 1 & 100,0 & 1,00 & $1,000(389)$ & 100,0 & - & - & - & - \\
2 & 63,4 & 1,60 & $0,766(508)$ & 50,6 & 359,4 & 9 & 56,5 & 77,3 \\
3 & 63,0 & 1,53 & $0,716(448)$ & 46,5 & 363,3 & 9 & 54,3 & 77,5 \\
4 & 36,5 & 2,70 & $0,460(517)$ & 15,1 & 623,1 & 22 & 31,5 & 57,9 \\
5 & 37,2 & 2,69 & $0,036(389)$ & 0,1 & 615,7 & 17 & 38,8 & 55,4 \\
6 & 25,9 & 3,76 & $0,019(508)$ & 0,3 & 726,9 & 19 & 20,5 & 48,0
\end{tabular}

Methode: 1: Idealfall (Originalwerte)

2: Siedlungsstatistik und -umrisse, alle Zellen

3: Siedlungsstatistik und -umrisse, minimale Flächenbedeckung einer Hektarzelle durch das Siedlungsgebiet: $10 \%$ (vgl. Text)

4: Siedlungsumrisse generalisiert, gleichförmige Verteilung der Bevölkerung

5: Bewohnte Zellen gemäß hektarweiser Auszählung, gleichförmige Verteilung der Bevölkerung

6: Siedlungsumrisse detailliert, gleichförmige Verteilung der Bevölkerung

A: Bestimmtheitsmaß ( $N=1646$, d. h. ganzes Gemeindegebiet) in \%

B: F-Wert: größere Varianz/kleinere Varianz bei Vergleich der Methode mit den Originalwerten

C: Spearman-Rangkorrelationskoeffizient (in Klammer die Anzahl der Beobachtungen); Zellen, die sowohl bei den Originaldaten aus auch bei der entsprechenden Methode den Wert $O$ enthalten, wurden ausgeschlossen

D: Bestimmtheitsmaß der Beobachtungspaare von $\mathrm{C}$ in \%

E: Varianz der Abweichungen von den Originalwerten $(N=565, d$. h. alle Zellen, die von mindestens einer Methode einen Wert $>0$ zugeteilt bekommen)

F: Interquartile Spannweite der Abweichungen ( $N=565)$

G: Abweichungen $\leq 5$ in $\%(N=565)$

$\mathrm{H}$ : Abweichungen $\leq 15$ in $\%(\mathrm{~N}=565)$

Tab. 2 Mittlere Distanzen und Bevölkerung in Einzugsgebieten

\begin{tabular}{|c|c|c|c|c|c|c|c|}
\hline \multirow[t]{2}{*}{ Methode $^{*}$} & \multicolumn{3}{|c|}{ Mittlere Distanz in $\mathrm{km}^{\star \star}$} & \multicolumn{4}{|c|}{ Bevölkerung in Distanzringen zum Gemeindehaus ${ }^{\star \star \star}$} \\
\hline & G & $\mathrm{T}$ & $\mathrm{B}$ & $0-300 \mathrm{~m}$ & $0-500 \mathrm{~m}$ & $0-1000 \mathrm{~m}$ & $0-1200 \mathrm{~m}$ \\
\hline 1 & 1,245 & 2,339 & 0,796 & 1174 & 2207 & 4446 & 5325 \\
\hline 2 & 1,232 & 2,340 & 0,800 & 1114 & 2141 & 4602 & 5282 \\
\hline 3 & 1,231 & 2,340 & 0,799 & 1143 & 2138 & 4604 & 5275 \\
\hline 4 & 1,298 & 2,372 & 0,854 & 812 & 1732 & 4128 & 4824 \\
\hline 5 & 1,370 & 2,212 & 0,965 & 737 & 1448 & 3659 & 4554 \\
\hline 6 & 1,400 & 2,145 & 1,037 & 618 & 1318 & 3420 & 4285 \\
\hline
\end{tabular}

* vgl. Tab. 1

** mittlere Distanz (Luftlinie, bevölkerungsgewichtet) vom Mittelpunkt der Rasterzellen zum Gemeindehaus (G), zum nächsten Bahnhof (B) und zum Restaurant Alpenblick im Weiler Toggwil (T)

*** vgl. Abb. 4

Abb. 4 Testgemeinde Meilen. Das eigentliche Wohngebiet ist schraffiert dargestellt. Die Distanzringe um das Gemeindehaus haben Radien von 300, 500, 1000 und 1200 Meter

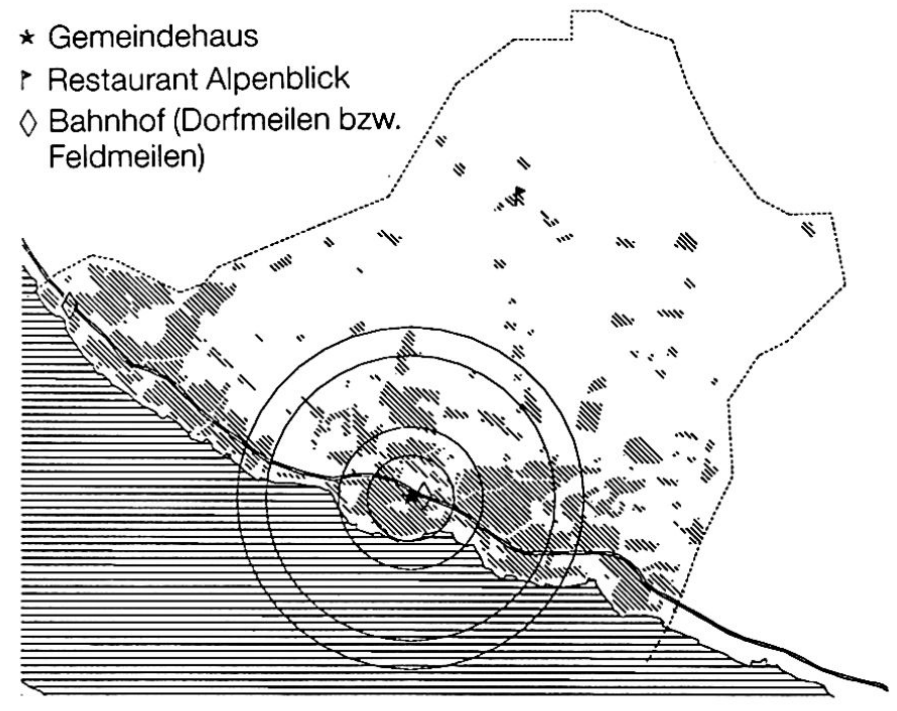




\section{Ergänzung der Siedlungsstatistik durch eigene Stichproben}

\subsection{Schätzung der Zahl der Privathaushaltungen}

Unbefriedigend blieben nach diesen Arbeiten die nicht mehr weiter aufgeteilten Siedlungen Dielsdorf (3636 Einw.), Erlenbach (4314 Einw.), Herrliberg (3834 Einw.) und Stäfa (5677 Einw.), das Quartier Männedorf-Dorfkern (4634 Einw.) sowie im Fall der Agglomeration alle außerkantonalen Gemeinden.

Als einziger Ausweg blieb die Möglichkeit, diese Gemeinden in Anlehnung an die bei der Zürcher Siedlungsstatistik verwendete Methode in Siedlungen und Quartiere zu unterteilen und zunächst die entsprechende Anzahl Haushaltungen zu schätzen. Als Hilfsmittel boten sich Telefonbuch und die Ortspläne mit Überdruck der Hausnummern an. Da diese Methode - soweit uns bekannt - noch nie angewendet wurde, soll sie hier etwas ausführlicher geschildert werden. In Anlehnung an FRIEDRICHS (1984, S. 129) können sechs nachzuprüfende Punkte bei der Arbeit mit Verzeichnissen unterschieden werden.

1. Ist das Verzeichnis vollständig oder gibt es a priori nicht erfaßte Gruppen?

2. Ist jedes Element vertreten?

3. Ist jedes Element nur einmal vertreten?

4. Sind Fremdelemente vorhanden?

5. Wie aktuell ist das Verzeichnis?

6. Wird die Reihenfolge der Elemente von bestimmten Eigenschaften beeinflußt?

Das Telefonbuch ist im geographischen Sinn für die entsprechene Region vollständig, umfaßt aber naturgemäß nur Privathaushaltungen mit Telefonanschluß. Die Schweiz weist traditionell eine sehr hohe Telefondichte auf, so daß namentlich in einem städtischen Raum wie der Agglomeration Zürich davon ausgegangen werden kann, daß praktisch jede Wohnung über einen Telefonanschluß verfügt. Vermutlich haben auch die meisten der zahlenmäBig kaum ins Gewicht fallenden Untermieterhaushalte einen eigenen Anschluß. In seltenen Einzelfällen, z. B. bei exponierten Persönlichkeiten, kann der an sich obligatorische Eintrag im Telefonbuch unterbleiben. Die in den ersten beiden Punkten geforderte Vollständigkeit ist somit beim Telefonbuch wenn nicht hundertprozentig, so doch im wesentlichen gewährleistet.

Die dritte Anforderung - jedes Element soll nur einmal vertreten sein - ist nicht erfüllt. Einträge mit mehreren Nummern lassen sich durch Ungültigerklärung aller zusätzlicher Nummern in den Normalfall überführen. Problematischer wird es, wenn mehrere Familien- (Ehepartner, Verwandte) oder Haushaltmitglieder (Wohngemeinschaften, unver- heiratete Paare) separat aufgeführt werden. Während die erste Kategorie noch relativ einfach eruierbar ist, kann die zweite nur mit unverhältnismäBigem Aufwand, nämlich mit einem Kreuzvergleich aller Telefonnummern, ausfindig gemacht werden. Völlig unmöglich ist die Identifikation von Zweitwohnungsanschlüssen. Die erwähnten «Doppelspurigkeiten» sind zwar in Zunahme begriffen, haben aber immer noch Ausnahmecharakter. Das gilt auch für die Zweitwohnungen (1980 1,6\% des gesamten Wohnungsbestandes in der Agglomeration Zürich, über 4\% nur in Stallikon; Kanton Zürich 1,7\% aller Wohnungen, höhere Werte nur in kleineren Gemeinden). Alle diese Fälle werden bei der Umsetzung der Methode deshalb vernachläßigt.

Fremdelemente sind im Telefonbuch in Form von Firmen, Kollektivhaushaltungen (z. B. Altersheimen) und ortsfremden Einträgen (z. B. Eintrag im Telefonverzeichnis einer Nachbargemeinde) ebenfalls vorhanden, doch sind diese meist unschwer zu erkennen. In unklaren Fällen liefern meist ein Pickup-Zeichen oder die Adresse Aufschluß darüber, ob es sich um einen Privat- oder einen Geschäftsanschluß handelt.

Bezüglich der Aktualität können Telefonbuch und Volkszählung nur annähernd zur Deckung gebracht werden, stimmt doch der Redaktionsschluß der Telefonbücher kaum je mit dem Zeitpunkt der Volkszählung (1. Dezember) überein. Im vorliegenden Fall bedeutet dies - unter Berücksichtigung einer Frist von drei bis fünf Monaten zwischen Redaktionsschluß und Publikation - gegenüber der Volkszählung eine Verspätung von rund einem halben Jahr (Gemeinden im Kanton Zürich, Band 15) bzw. einen Vorsprung von nicht ganz einem halben Jahr (Gemeinden im Kanton Aargau, Band 11). Einzig bei der Gemeinde Wollerau (Band 13) stimmt der Redaktionsschluß ziemlich genau mit dem Zeitpunkt derVolkszählung 1980 überein. Bei einer starken Bautätigkeit muß also mit gewissen Abweichungen gerechnet werden. Dennoch dürfte das Telefonbuch einen weit besseren Rückschluß auf die Zahl der Privathaushaltungen erlauben als das Adreßbuch, das allein schon durch sein Erfaßungsprinzip (alle erwachsenen Personen mit Ausnahme der verheirateten Frauen) kaum lösbare Probleme stellt.

\section{2 Überprüfung der Methode}

Anhand von zwei Testgemeinden - Dällikon und Oberglatt - wurde die Methode auf ihre Tauglichkeit überprüft (vgl. Tab. 3a und $3 \mathrm{~b}$ ). Insbesondere sollte auch abgeklärt werden, ob das Telefonbuch als Grundgesamtheit für eine Stichprobe verwendet werden kann. Aus arbeitstechnischen Gründen wurden zwei Gemeinden ausgewählt, die für die Siedlungsstatistik in mehrere größere Ortsteile aufgegliedert worden sind ${ }^{2}$, für die aktuelle Hausnummernpläne existieren und deren Größe eine voll- 


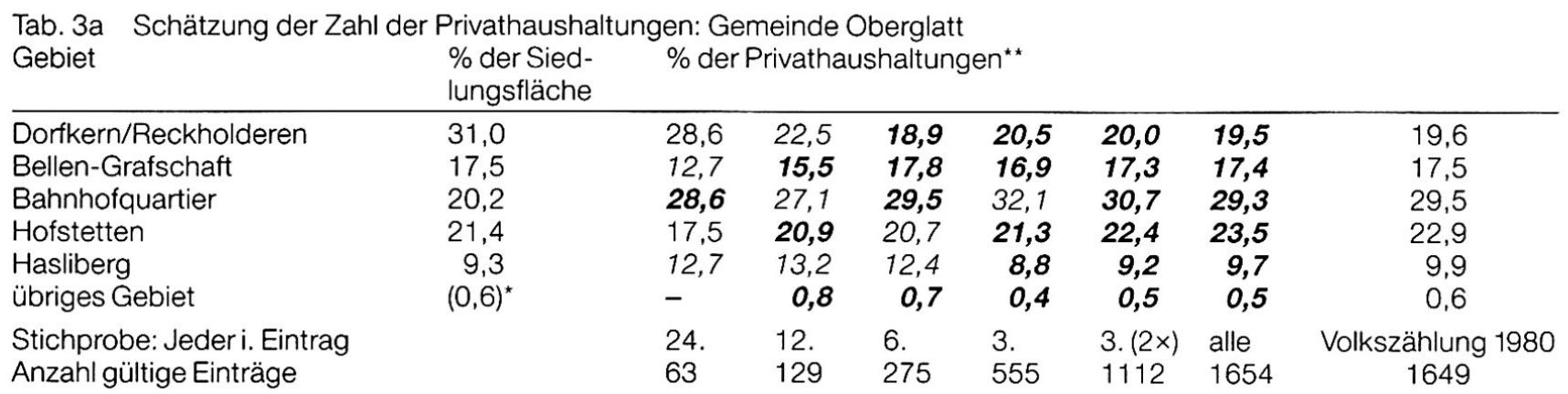

Tab. 3b Schätzung der Zahl der Privathaushaltungen: Gemeinde Dällikon

\begin{tabular}{|c|c|c|c|c|c|c|}
\hline Gebiet & $\begin{array}{l}\% \text { der Sied- } \\
\text { lungsfläche }\end{array}$ & $\%$ der & Privatha & shaltun & $e n^{\star \star}$ & \\
\hline Dorf & 54,8 & 64,6 & 55,9 & 56,0 & 54,8 & 54,7 \\
\hline Rain & 17,4 & 4,2 & 7,5 & 8,9 & 10,9 & 12,3 \\
\hline Seitenacker & 19,3 & 20,8 & 24,7 & 22,5 & 19,6 & 18,3 \\
\hline Industriestraße & 4,5 & 8,3 & 10,8 & 8,4 & 11,1 & 10,8 \\
\hline übriges Gebiet & $(4,0)^{\star}$ & 2,1 & 1,1 & 3,7 & 3,5 & 4,0 \\
\hline $\begin{array}{l}\text { Stichprobe: jederi. Eintrag } \\
\text { Anzahl qültige Einträge }\end{array}$ & & $\begin{array}{l}20 . \\
48\end{array}$ & $\begin{array}{l}10 . \\
93\end{array}$ & $\begin{array}{l}5 . \\
191\end{array}$ & $\begin{array}{l}\text { alle } \\
911\end{array}$ & $\begin{array}{c}\text { Volkszählung } 1980 \\
885\end{array}$ \\
\hline
\end{tabular}

* Nicht berechnet, sondern dem aus der Siedlungsstatistik bekannten Bevölkerungsanteil gleichgesetzt.

** kursiv: im 5\%-Bereich/halbfett kursiv: im 2\%-Bereich

ständige Lokalisierung und Auszählung aller Telefonbucheinträge mit vertretbarem Zeitaufwand noch gestattet.

In beiden Tabellen kommt die mit der Stichprobengröße wachsende Annäherung an die Volkszählungsresultate deutlich zum Ausdruck. Die vollständige Auszählung erbringt im Fall von Oberglatt Resultate, die fast identisch mit der Volkszählung sind. Die einzige nennenswerte Abweichung erreicht bloß 0,6 Prozentpunkte, was 12 «überzähligen» Adressen entspricht. Im Fall von Dällikon sind die Abweichungen generell etwas größer, bleiben aber mit maximal 1,3 bzw. 1,4 Prozentpunkten (entsprechend 10 Einträgen zuwenig oder 16 Einträgen zuviel) ebenfalls noch nahe an den Volkszählungswerten. Arbeitstechnisch von Bedeutung ist die Annäherung der Stichprobenergebnisse an die Vollauszählung. Schon bei der Stichprobengröße von etwa 200 läßt sich die räumliche Verteilung der Privathaushalte wesentlich genauer schätzen als mit einer flächenproportionalen Disaggregation ${ }^{3}$ (vgl. Tabellen $3 a$ und $3 b$ ). Einen besonderen Wert erhält die Methode dadurch, daß die Zahl der Privathaushaltungen mit relativ geringem Aufwand für beliebig abgrenzbare Gebiete recht genau geschätzt werden kann. Durch die Verwendung des Telefonbuchs sind solche Schätzungen zudem fast für jedes Stichjahr möglich und nicht nur für die Volkszählungsjahre.

Da die außerhalb des Kantons Zürich gelegenen Agglomerationsgemeinden keine vergleichbare amtliche Siedlungsstatistik kennen, mußten in diesen Fällen zuerst alle in Frage kommendenTelefonbuch- einträge nach Adressen außerhalb der Hauptsiedlung durchgesehen werden. Für jede gefundene Adresse wurden eine Privathaushaltung bzw. drei Einwohner in Privathaushalten vom Gemeindetotal subtrahiert ${ }^{4}$. Für die drei kleinsten Aargauer Gemeinden (Killwangen, Arni und Islisberg), die nicht mehr weiter unterteilt werden sollten, war nach diesem Schritt die Bevölkerungsschätzung beendet.

Tabelle 4 gibt Auskunft über die Zahl der für die eigentliche Disaggregation übrigbleibenden Privathaushaltungen der restlichen zu disaggregierenden Gemeinden bzw. Gemeindeteile. Gemeinden mit weniger als 500 Haushalten wurden vollständig ausgezählt.

\subsection{Schätzung der Einwohnerzahl}

Der Rückschluß von der Zahl der Privathaushaltungen auf die Zahl der Einwohner in diesen Haushaltungen ist nicht trivial, da die Haushaltgröße je nach Gebiet beträchtlich schwanken kann. Insbesondere weisen neuere Einfamilienhausgebiete i. a. wesentlich höhere durchschnittliche HaushaltgröBen auf als Altbaugebiete. Um diesem Umstand Rechnung zu tragen, wurden verschiedene Variablen in den im Kanton Zürich gelegenen Agglomerationsgemeinden darauf untersucht, ob sie mit der Haushaltgröße in den 740 Siedlungen, Quartieren und (in der Stadt Zürich) Statistischen Zonen mit mindestens 50 Einwohnern korrelieren. Dabei standen formale Größen, d. h. solche, die durch Karteninterpretation oder Feldbegehung schätzbar 
Tab. 4 Grundgesamtheiten und Stichprobengrößen in den zu disaggregierenden Gemeinden

\begin{tabular}{|c|c|c|c|c|c|c|c|c|c|}
\hline \multirow[t]{2}{*}{ Gemeinde } & \multicolumn{3}{|c|}{ Privathaushalte } & \multicolumn{3}{|c|}{ Personen in Privathaushalten } & \multicolumn{3}{|c|}{ Telefonbuchstichprobe } \\
\hline & $\mathrm{t}$ & a & ü & $\mathrm{t}$ & a & $\ddot{u}$ & Eintrag & total & Einträge \\
\hline Wollerau & 1350 & 128 & 1222 & 3861 & 384 & 3477 & 5. & 259 & 235 \\
\hline Bergdietikon & 550 & 72 & 478 & 1681 & 218 & 1463 & alle & 563 & 491 \\
\hline Spreitenbach & 2895 & 33 & 2862 & 7129 & 99 & 7030 & 12. & 227 & 224 \\
\hline Berikon & 765 & 13 & 752 & 2304 & 39 & 2265 & 3. & 246 & 242 \\
\hline Oberwil-Lieli & 386 & 14 & 372 & 1191 & 42 & 1149 & alle & 389 & 375 \\
\hline Rudolfstetten & 1312 & 9 & 1303 & 3582 & 27 & 3555 & 5. & 250 & 248 \\
\hline Widen & 924 & 16 & 908 & 2655 & 48 & 2607 & 4. & 223 & 221 \\
\hline Zufikon & 884 & 19 & 865 & 2613 & 57 & 2556 & 4. & 209 & 202 \\
\hline Dielsdorf & 1291 & 60 & 1231 & 3434 & 160 & 3274 & 6. & 227 & 208 \\
\hline Erlenbach & 1681 & 18 & 1663 & 4038 & 54 & 3984 & 7. & 255 & 246 \\
\hline Herrliberg & 1454 & - & 1454 & 3593 & - & 3593 & 7. & 250 & 225 \\
\hline Männedorf-Dorfk. & 1752 & - & 1752 & 4284 & - & 4284 & 8. & 367 & 212 \\
\hline Stäfa & 2225 & - & 2225 & 5465 & - & 5464 & 10. & 327 & 231 \\
\hline
\end{tabular}

t: total Nolkszählung 1980)

a: außerhalb der Hauptsiedlung (Schätzung aufgrund der Telefonbucheinträge)

ü: für die Disaggregation übrigbleibend

sind, im Vordergrund: Hausbesitz, Alter der Wohnung (Anteil der Bauperiode a) vor 1900, b) $1900-1920$, c) $1921-1946$, d) $1947-1960$, e) $1961-1970$, f) $1971-1975$ und g) 1976-1980), wichtigste Bauperiode, Bauperiode, in der k) $50 \%$, l) $75 \%$ des heutigen Wohnungsbestandes erreicht wurden, Gebäudeart (Anteil der Wohungen im 3. und höheren Stockwerken).

Nach einer schrittweisen multiplen Regression konnte die Auswahl der Variablen auf die Prozentanteile der Hauseigentümer, der Wohnungen aus der Zeit vor 1947 sowie der Wohnungen aus der Bauperiode 1947-1960 eingeschränkt werden. Diese drei Variablen erklären 63\% der Streuung der durchschnittlichen Haushaltsgrößen. Eine Gewichtung der Siedlungen nach Größe wurde bewußt unterlassen, da die größeren Einheiten naturgemäß weniger homogen sind und zum Gesamtmittel tendieren.

Setzt man als Randbedingung fest, daß in jeder Gemeinde die Summe der geschätzten Bevölkerungszahlen der Aggregate der - bekannten - Bevölkerungszahl der zu disaggregierenden Gemeinde entsprechen muss ${ }^{5}$, nimmt die erklärte Streuung auf $71 \%$ zu. Eine Ausklammerung der naturgemäß eher zu Extremwerten neigenden kleinsten Siedlungen (d. h. derjenigen mit 50 bis 200 Einwohnern) bringt eine weitere Verbesserung auf $80 \%$. Da alle angestrebten Bevölkerungsschätzungen auf Vorortsgemeinden entfallen, ist es angezeigt, die Stadt Zürich von der Regressionsrechnung auszunehmen. Weil das Modell im Fall der Statistischen Zonen der Stadt Zürich noch um eine Spur bessere Resultate liefert als für die Siedlungen und Quartiere der übrigen Agglomerationsgemeinden, sinkt der
Anteil der erklärten Varianz bei den übrigbleibenden 345 Beobachtungen auf $75 \%$. Die dazugehörige Regressionsrechnung lautet:

$\mathrm{HHG}^{\wedge}=-0,52 \times \mathrm{BP} 1-0,33 \times \mathrm{BP} 2+1,06 \times \mathrm{HEI}+2,54$

wobei $\mathrm{HHG}^{\wedge}$ : geschätzte Haushaltgröße

BP1: Anteil vor 1947 erstellte Wohnungen BP2: Anteil 1947-1960 erstellten Wohnungen

HEI: Hauseigentümer in \% aller Haushaltungen

Zur Kontrolle wurde dieses Verfahren wiederum auf die Testgemeinden Dällikon und Oberglatt angewendet (Tabellen 5a und 5b)

Bezogen auf die Gesamtbevölkerung, weichen alle Schätzungen weniger als $2 \%$ von den Volkszählungsergebnissen $a b$, im Fall von Oberglatt sogar weniger als $1,5 \%$. Damit darf die Brauchbarkeit der Methode als gesichert gelten.

Einen visuellen Eindruck der durch die Daten der Siedlungsstatistik und die Ergebnisse der eigenen Stichproben vervollständigten Hektarrasterdatei vermittelt Abbildung 5. Im Bereich dieses Kartenausschnittes sind alle erwähnten Arbeitsschritte zur Anwendung gelangt. Dennoch fällt es dem unvoreingenommenen Auge schwer, große Unterschiede festzustellen. Die gute optische und statistische Übereinstimmung ist im Hinblick auf die Volkszählung 1990 nicht ganz unbedeutend, zeigt sie doch, daß eine zweckmäßige Siedlungsstatistik bis zu einem gewissen Grad durchaus ein Ersatz für die wesentlich aufwendigere hektarweise Erfassung sein kann. 
Tab. 5a Ergebnisse der Bevölkerungsschätzung für die Gemeinde Oberglatt

$\begin{array}{lcccrr}\text { Gebiet } & \begin{array}{l}\text { Anzahl Privathaush. } \\ \text { geschätzt (Volkszähl.) }\end{array} & \begin{array}{l}\text { geschätzte } \\ \text { Haushaltgröße }\end{array} & \begin{array}{l}\text { Korrektur- } \\ \text { faktor }\end{array} & \begin{array}{l}\text { Bevölkerung in Privathaushalten } \\ \text { geschätzt }\end{array} & \begin{array}{r}\text { Volkszählung } \\ \text { Dorfkern/Reckholderen }\end{array} \\ \text { Bellen-Grafschaft } & 281(323) & 2,60 & 0,958 & 801 & 845 \\ \text { Bahnhofquartier } & 482(489) & 2,57 & 0,958 & 693 & 636 \\ \text { Hofstetten } & 386(377) & 2,58 & 0,958 & 1173 & 1194 \\ \text { Hasliberg } & 160(163) & 2,57 & 0,958 & 953 & 987 \\ \text { übriges Gebiet } & -(10) & 2,59 & 0,958 & 395 & 353 \\ \text { total } & (1649) & - & - & (38) & (38) \\ & & - & - & 4053 & 4053\end{array}$

Tabelle 5b Ergebnisse der Bevölkerungsschätzung für die Gemeinde Dällikon

$\begin{array}{lcccrr}\text { Gebiet } & \begin{array}{c}\text { Anzahl Privathaush. } \\ \text { geschätzt (Volkszähl.) }\end{array} & \begin{array}{l}\text { geschätzte } \\ \text { Haushaltgröße }\end{array} & \begin{array}{l}\text { Korrektur- } \\ \text { faktor }\end{array} & \begin{array}{l}\text { Bevölkerung in Privathaushalten } \\ \text { geschätzt }\end{array} & \begin{array}{r}\text { Volkszählung } \\ \hline \text { Dorf }\end{array} \\ \text { Industriestraße } & 480(484) & 2,59 & 0,996 & 1238 & 1255 \\ \text { Seitenacker } & 97(109) & 2,59 & 0,996 & 252 & 261 \\ \text { Rain } & 172(162) & 2,74 & 0,996 & 469 & 431 \\ \text { übriges Gebiet } & 95(96) & 3,43 & 0,996 & 326 & 338 \\ \text { total } & -(35) & - & - & (95) & (95) \\ & 885 & - & - & 2380 & 2380\end{array}$

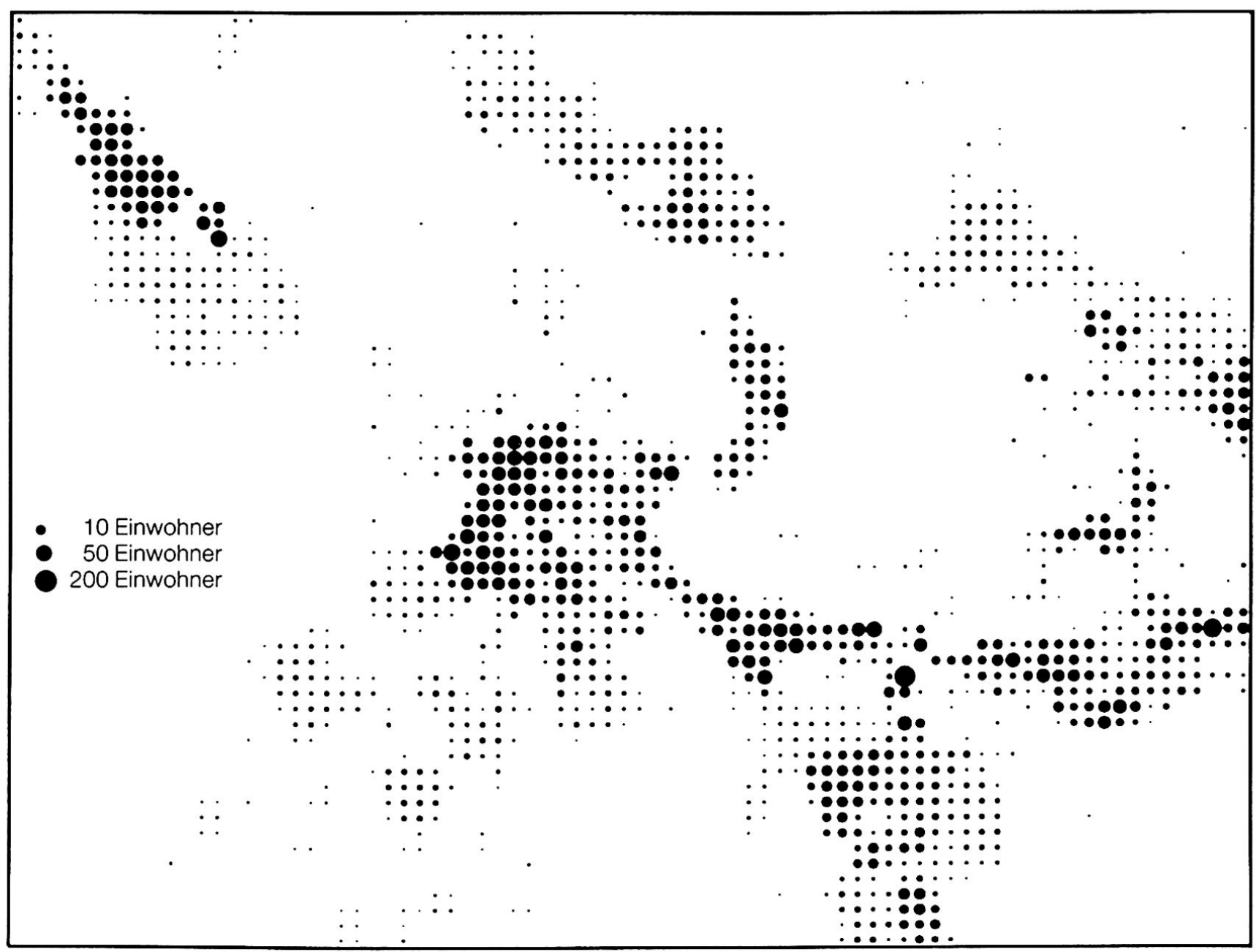

Abb. 5 Ausschnitt aus der ergänzten Hektarrasterdatei (Beispiel Limmattal). In der Mitte der Abbildung liegt Dietikon, links oben Spreitenbach, rechts oben Unterengstringen. Die Fläche der Punktsignaturen ist proportional zur Bevölkerungszahl der einzelnen Hektarzellen. 


\section{Anmerkungen}

$1 \mathrm{H}$. WINDLER und E. WINKLER, Zur quantitativen Bestimmung von Siedlungseinheiten. In: Plan und Ortsplanung. Heft 6/1950, S. $180 \mathrm{ff}$.

2 Bei nur wenigen unterschiedlich großen Einheiten (z. B. $90 \%$ in einem und $10 \%$ in einem anderen Gebiet) sind die Abweichungen, bezogen auf das größere Gebiet, stets relativ klein.

3 Der Vertrauensbereich von Stichproben-Resultaten hängt nur von der Größe der Stichprobe, nicht aber derjenigen der Grundgesamtheit ab (FRIEDRICHS, 1984, S. 145). Bei 5\% Irrtumswahrscheinlichkeit genügt auch im ungünstigsten Fall für einen Vertrauensbereich von $\pm 10 \%$ eine Stichprobengröße von 96, während für einen Vertrauensbereich von $\pm 1 \%$ bereits eine solche von 9600 nötig wäre; eine Stichprobengröße von 200 entspricht einem Vertrauensbereich von $\pm 7 \%$.

${ }^{4}$ Möglichenweise ist dies eine Unterschätzung, aber im Hinblick auf eine Hektarzuordnung die einzige Möglichkeit, da ganzzahlige Werte erfordert sind. Einzige Ausnahme ist die Gemeinde Dielsdorf, wo für eine isolierte Blocksiedlung eine Haushaltgröße von 2,5 eingesetzt wurde.

5 Erreicht durch gemeindeweise Multiplikation mit dem Quotienten von tatsächlichem und geschätztem Gemeindetotal. Als Gleichung für die Bevölkerungsschätzung ergibt sich unter Einbau des Korrekturfaktors:

$B E{ }^{\wedge}{ }_{j}=\left(B E V / \Sigma B E{ }^{\wedge}{ }_{j}\right) \times\left(H H G^{\wedge}{ }_{j} \times H H \times Q_{i} / \Sigma Q_{i}\right)$

wobei: $\quad \mathrm{BEV}^{\wedge}$ : geschätzte Bevölkerung im Teilgebiet $\mathrm{i}$

BEV: Gesamtbevölkerung der Gemeinde x

$\mathrm{HHG}^{\wedge}{ }_{\mathrm{i}}$ : geschätzte Haushaltsgröße im Teilgebiet $\mathrm{i}$

$\mathrm{HH}$ : Privathaushaltungen der Gemeinde $x$

$Q_{i}:$ Größe der Stichprobe des Teilgebiets i

\section{Literatur}

ACKERKNECHT, D. (1969): Informationsraster (landesplanerische Datenbank), 1. und 2. Teil: Übersicht und Vorbereitungsarbeiten, Arbeitsberichte zur ORL, Nr. 4.1 und 4.2, Februar 1969

BUNDESAMT FÜR STATISTIK (1980 ff.): Informationsraster Benützerhandbuch, Arbeitsdokumente für die Schweizer Statistik, Heft 3, 1980; diverse Nachträge

FRIEDRICHS, J. (1984): Methoden empirischer Sozialforschung, 12. Auflage, Opladen

HERZOG, A. und WEIBEL R. (1985 ff.): GRID/GRIDUNI Benutzerhandbuch, Manuskript, Geogr. Institut der Universität Zürich

INFRAPLAN (o.J.): Hektarweise Ermittlung der Bevölkerungsverteilung in verschiedenen Regionen der Schweiz, Manuskript, Wabern-Bern.

STATISTISCHES AMT DES KANTONS ZÜRICH (1985): Siedlungen und Gemeinden des Kantons Zürich, Statistische Mitteilungen des Kantons Zürich, Heft 114, Dritte Folge, Mai 1985 